\title{
Seroprevalencia de los Virus de las Hepatitis A y B en Grupos Etarios de Costa Rica
}

\author{
Mayra L. Taylor, ${ }^{1}$ Zaida García, ${ }^{1}$ lleana Holst, ${ }^{2}$ Teresita Somogye,${ }^{2}$ Lowella Cunningham, ${ }^{3}$ Kirsten A. Visoná ${ }^{1}$
}

\begin{abstract}
Justificación y Objetivos: Debido a la persistencia de casos y brotes de hepatitis viral A y B en el país, junto con la observación de un aumento importante de infecciones por el tipo A en la población de adultos, se planteó la necesidad de establecer la prevalencia de estos dos tipos de hepatitis en diferentes grupos etarios en nuestro país.
\end{abstract}

Métodos: La seroprevalencia de los virus de la hepatitis A (VHA) y de hepatitis B (VHB), fueron investigadas en 873 muestras de niños pre-escolares menores de 7 años de la encuesta nacional de nutrición en 1996; 614 niños escolares de 9 a 15 años de una encuesta de nutrición de la Universidad de Costa Rica realizada en 1997 y 996 muestras de adultos, estudiantes y funcionarios de esta misma universidad recolectadas en 1994. Las muestras se analizaron por anticuerpos totales contra el VHA (anti-VHA total), VHB antígeno de superficie (HBsAg) y anticuerpos totales contra la proteína del núcleo o "core" (anti-HBc total).

Resultados: Se encontró un $6.8 \%, 13.2 \%$ y $71.7 \%$ de anti-VHA total en los preescolares, escolares y adultos, respectivamente, con una creciente prevalencia en relación con la edad. La presencia de estos anticuerpos en los escolares estuvo asociada con la escolaridad de los padres y su condición socioeconómica; siendo mayor cuando los padres tenían un menor nivel de escolaridad ( $24 \%$ con secundaria incompleta versus $7.6 \%$ con nivel educativo superior) y entre padres sin capacitación técnica o profesionales (16\% versus $8.7 \%$ en los padres con estudios superiores). También se encontró una diferencia en la prevalencia de anti-VHA entre estudiantes de escuelas públicas $(19.5 \%)$ versus privadas (6.9\%). En los preescolares, el único grupo con representación estadística geográfica; la provincia de Guanacaste presentó un riesgo de 2 a 3 veces mayor de tener anticuerpos contra el VHA (21\%) que los niños procedentes de otras provincias, lo cual es concordante con los datos relacionados con condiciones socioeconómicas más bajas, falta de agua potable, letrinización y hacinamiento en las áreas rurales.

En cuanto a los marcadores de VHB, se encontró una prevalencia de $0.5 \%, 0.2 \%$ y $0.2 \%$ para HBsAg y $2.8 \%$, $0.3 \%$ y $4.0 \%$ para el anti-HBc total en preescolares, escolares y adultos, respectivamente. No hubo ninguna relación entre las condiciones socioeconómicas o la edad, para estas prevalencias, pero el anti-HBc fue más alto en los hombres (6.9\% en hombres y $2.3 \%$ en mujeres).

Conclusiones: Al comparar los datos obtenidos en décadas anteriores en Costa Rica sobre estos dos tipos de hepatitis se encontró que la hepatitis $\mathrm{B}$, sigue teniendo un patrón epidemiológico similar de baja prevalencia, mientras que la hepatitis A presentó un cambio de patrón durante la infancia, al pasar de una prevalencia alta a una intermedia a baja. Este cambio requiere de una intervención oportuna para evitar brotes epidémicos y un aumento en el número de casos severos en jóvenes y adultos.

Recibido: 4 de julio de 2001.

Aceptado: 23 de octubre de 2001.

\footnotetext{
Centro Internacional de Investigación y Adiestramiento Médico de la Universidad del Estado de Louisiana (LSU-CIIAM).

Facultad de Microbiología, Universidad de Costa Rica, UCR.

3 Instituto Costarricense de Investigación y Enseñanza en Nutrición y Salud (INCIENSA)
}

Correspondencia: M. Lizeth Taylor C. SJO 321, 1601 N.W. 97Th Ave, P. O. Box 10155-1000 San José. Fax: (506) 279 3119. Correo electrónico: lizethtaylor@hotmail.com

\section{Introducción}

Los virus de hepatitis A y B durante la fase aguda de la infección, pueden causar enfermedad clínica y casos fulminantes o permanecer como infección asintomática. Los dos tipos de virus pueden producir cuadros agudos semejantes con inflamación del hígado, alteración de los marcadores bioquímicos de función hepática, malestar general, vómitos, anorexia, fatiga, heces de color claro (hipocolia o acolia), orina oscura (coluria) y en algunos casos ictericia. ${ }^{1,2}$ 
El virus de la hepatitis A, se identificó en 1973, y se clasificó como un picornavirus del género de los hepatovirus. ${ }^{3} \mathrm{Su}$ transmisión se produce por vía fecal-oral, se asocia con el consumo de agua y alimentos contaminados, contacto de persona a persona y se relaciona con el nivel sanitario e higiénico de una población. ${ }^{1,4}$ Se han reportado infecciones nosocomiales principalmente en recién nacidos de unidades de cuidados intensivos, guarderías infantiles ${ }^{5,6}$ y más recientemente infección causada por transfusión de derivados sanguíneos. ${ }^{7,8}$

La hepatitis A, transcurre en gran parte desapercibida en niños. En adolescentes y adultos la enfermedad es más severa y se presentan aproximadamente un $2 \%$ de casos fulminantes. ${ }^{1}$ Este virus no produce cronicidad y los anticuerpos contra VHA tipo IgG persisten por largo tiempo otorgando inmunidad contra todos los genotipos. ${ }^{9}$

Se consideran países de alta prevalencia por Virus de Hepatitis A (VHA) cuando el 30\%-40 \% de niños menores de 5 años y el 70\%-100\% de adolescentes tienen anticuerpos, prevalencia intermedia con $10 \%-25 \%$ en menores de 5 años y aproximadamente $50 \%$ en personas mayores de 15 años y prevalencia baja con porcentajes menores a los indicados anteriormente. ${ }^{10}$ Costa Rica se encontraba documentada hasta ahora entre los países con una alta prevalencia de infección por VHA. ${ }^{11}$

El mejoramiento de las condiciones sanitarias en una comunidad o población retarda la exposición al VHA y por consiguiente la posibilidad de adquirir la infección, reduciendo así la prevalencia en niños y aumentando el riesgo en adultos. ${ }^{12-14}$ En estudios realizados por el Centro Internacional de Investigación y Adiestramiento Médico (CIIAM) en los últimos 10 años, se observa un aumento en la morbilidad por VHA en jóvenes y adultos. Un estudio de 108 estudiantes de medicina de la Universidad de Costa Rica, mostró que el 60\% de esta población era susceptible a la infección por VHA, lo cual sugiere que en Costa Rica se está estableciendo un patrón intermedio de infección y con un riesgo substancial para que adultos adquieran la infección y padecer la enfermedad (Datos no publicados del CIIAM).

Las estrategias aplicadas durante las últimas décadas para el control del VHA consistían en el mejoramiento ambiental, control microbiológico y la correcta manipulación de los alimentos, ninguna de las cuales es efectiva individualmente. ${ }^{10}$ En la actualidad se dispone; además, de vacunas que pueden brindar una herramienta efectiva para el control de brotes y epidemias y eventual erradicación de la enfermedad. Sin embargo, el costo elevado de las mismas, hacen inaccesibles campañas de vacunación masiva en los países en desarrollo. ${ }^{12,15,16}$

El virus de la hepatitis B (VHB), es un miembro de la familia Hepadnaviridae. Se transmite principalmente por vía parenteral, sexual, perinatal y por contacto intrafamiliar. El VHB es causante de portadores crónicos los cuales se determinan por la circulación del HBsAg en la sangre por más de 6 meses. ${ }^{2}$ El riesgo de una población de infectarse con el VHB depende de la prevalencia de portadores crónicos, genotipos circulantes y la práctica de hábitos de riesgo. ${ }^{17,18}$ Se estima que actualmente existen entre 250 y 350 millones de personas portadoras crónicas del VHB en el mundo. ${ }^{2,19}$

El porcentaje de cronicidad producido por el VHB varía según la edad de adquisición de la enfermedad.,20 Cuando la infección es adquirida por vía perinatal el riesgo de desarrollo de cróniciad es de hasta un $90 \%$; si la infección ocurre durante los primeros 5 años de vida el riesgo es del 30\%-40\%, y de 5\%-10\% si se adquiere después de los 5 años. El estado de cronicidad neonatal desarrollado durante la niñez tiene un mayor riesgo de evolucionar a enfermedad hepática severa a temprana edad como cirrosis y/o cáncer, con una alta tasa de mortalidad. ${ }^{21}$

La prevalencia del VHB varía según las diferentes zonas geográficas y distribución demográfica. ${ }^{19,22,23}$ Se consideran zonas de alto riesgo cuando la prevalencia de portadores supera el $8 \%$, de riesgo intermedio con prevalencia de $2 \%-8 \%$ y de bajo cuando la prevalencia es menor del $2 \%$. El contacto previo con el VHB evidenciado por la presencia de anti-core, es de 5 a 15 veces mayor que la prevalencia de HBsAg de portadores crónicos. ${ }^{24}$

Estudios seroepidemiológicos realizados por el CIIAM en donantes de sangre, del área metropolitana, así como también de las zonas de San Carlos, Pérez Zeledón, San Vito de Coto Brus y San Ramón-Palmares, demostraron que Costa Rica puede ubicarse dentro de una región con un patrón de baja endemicidad para la hepatitis B (0.5\%-1\%), con excepción de Pérez Zeledón donde la prevalencia es intermedia $(2 \%-3 \%) .{ }^{25,26}$

Los programas de vacunación contra la hepatitis B durante las dos últimas décadas han tenido gran impacto en la prevención y control del VHB. ${ }^{27,28}$

El objetivo del presente estudio fue conocer en diferentes grupos etarios del país, la prevalencia de inmunidad contra el VHA y de infección presente o pasada por el VHB, con el fin de que estos resultados permitan ayudar a las autoridades pertinentes a diseñar planes estratégicos adecuados de prevención, vacunación y respuesta a brotes epidémicos.

\section{Materiales y Métodos}

Población en estudio: Se analizaron 873 muestras de sangre de niños preescolares (menores de 7 años) del banco de suero de la Encuesta Nacional de Nutrición, con representación estadística, efectuada por el Ministerio de Salud durante 1996; 614 muestras de niños de 9 a 15 años del banco de sueros de una encuesta nutricional metropolitana de la Universidad de Costa Rica realizada en 1997; y muestras de 996 estudiantes y funcionarios de la Universidad de Costa Rica que asistieron a servicios de salud durante 1994. 
Recolección de la información: Se usaron las bases de datos existentes (Ministerio de Salud. Encuesta Nacional de Nutrición. 1 Fascículo Antropometría. Costa Rica. 1996. Ministerio de Salud, INCIENSA) para los niños $<7$ años que incluyeron datos de edad, sexo, lugar de residencia, escolaridad, ocupación de los padres y condición de vivienda.

Del grupo de los escolares de 9-15 años, la base de datos fue proporcionada por la Universidad de Costa Rica, con la siguiente información: edad, sexo, lugar de residencia, tipo de escuela, nivel escolar del niño, escolaridad de los padres, ocupación y condición y tenencia de vivienda.

Finalmente, se recolectó la información pertinente de estudiantes y funcionarios de los expedientes de la Oficina de Salud de la Universidad de Costa Rica, mediante el uso de un cuestionario diseñado para tal efecto. Se incluyeron datos de edad, sexo y lugar de residencia.

Metodología: Para la determinación de anti-VHA se usó un reactivo comercial de los Laboratorios Abbott, Chicago, IL., EIA-HAVAB y/o el RIA anti-HAV desarrollado por el CIIAM. Los dos métodos se basan en una metodología competitiva previamente descrita. Brevemente, $5 \mu 1$ de muestra se pone a competir en $95 \mu 1$ de un anti-HAV marcado con ${ }^{125} \mathrm{I}$ previamente titulado por el virus de hepatitis A capturado en una placa de poliestireno. Se incuba durante 2 horas a $40^{\circ} \mathrm{C}$, se lava y se leen en las cuentas por minuto en un contador gamma (1470 Wizard, Wallac). Se considera una reacción positiva aquella donde se disminuya igual o más del $50 \%$ de la lectura promedio de los controles positivos y negativos. ${ }^{29,30}$ Reactivos comerciales de los laboratorios Abbott, Chicago, fueron utilizados para detección de HBsAg (Auszyme), antiHBc (EIA-Corab) y/o reactivos desarrollados por CIIAM, RIA-HBsAg y RIA-anti-HBc. Los métodos comerciales y del CIIAM fueron basados en metodologías semejantes. Se usó el principio de sandwich simple para el HBsAg y de competencia para el anti-HBc. ${ }^{31}$ Para la detección de HBsAg, $100 \mu 1$ de muestra se incuban durante una hora en una placa recubierta con anticuerpos anti-HBsAg (anti-HBs), se lavan y se incuban por 2 horas con un anti-HBs marcado con ${ }^{125} \mathrm{I}$. Se lava y se lee en un contador gamma. Se considera positiva toda muestra con valores 2.1 veces superiores al promedio de los controles negativos. El principio de la prueba de anti-HBc es similar al anteriormente descrito para el anti-HAV usando como antígeno una proteína "core" recombinante. ${ }^{31}$

\section{Resultados}

El Cuadro 1, muestra la distribución por provincia de las tres poblaciones del estudio, reflejando la falta de muestras obtenidas en el grupo escolar en las provincias de Guanacaste, Puntarenas y Limón.

La prevalencia de anticuerpos contra el VHA mostró una variación importante entre las tres poblaciones, con una notable tendencia a aumentar de manera proporcional con la edad (Cuadro 2). Al subdividir el grupo escolar en dos categorías

\section{Cuadro 1: Distribución por provincia de pre-escolares, escolares y adultos}

\begin{tabular}{lccccccc}
\hline & \multicolumn{3}{c}{ Preescolares } & \multicolumn{2}{c}{ Escolares } & \multicolumn{2}{c}{ Adultos } \\
Provincia & $\mathrm{N}^{\circ}$ & $\%$ & $\mathrm{~N}^{\circ}$ & $\%$ & $\mathrm{~N}^{\circ}$ & $\%$ \\
\hline San José & 327 & 37.5 & 569 & $94-4$ & 566 & 74.9 \\
Alajuela & 126 & 14.4 & 1 & 0.2 & 48 & 6.3 \\
Cartago & 62 & 7.1 & 13 & 2.2 & 67 & 8.9 \\
Heredia & 55 & 6.3 & 20 & 3.3 & 52 & 6.9 \\
Guanacaste & 91 & 10.4 & - & - & 10 & 1.3 \\
Puntarenas & 127 & 14.5 & - & - & 6 & 0.8 \\
Limón & 85 & 9.7 & - & - & 7 & 0.9 \\
\hline Total & 873 & 100 & 603 & 100 & 756 & 100 \\
\hline
\end{tabular}

\section{Cuadro 2: Prevalencia de anti-VHA total, HBsAg $y$ anti-HBc total en preescolares, escolares y adultos de Costa Rica, 1994-1997}

\begin{tabular}{lcccc}
\hline Población & Total & $\begin{array}{l}\text { Anti-VHA total } \\
\text { Positivos \% }\end{array}$ & $\begin{array}{l}\text { HBsAg } \\
\text { Positivos \% }\end{array}$ & $\begin{array}{l}\text { Anti-HBc total } \\
\text { Positivos \% }\end{array}$ \\
\hline $\begin{array}{l}\text { Pre-escolares } \\
<7 \text { años }\end{array}$ & 873 & 6.8 & 0.5 & 2.8 \\
$\begin{array}{l}\text { Escolares } \\
\begin{array}{l}\text { 9-15 años } \\
\text { Adultos UCR } \\
\text { (estudiantes y } \\
\text { funcionarios) }\end{array}\end{array}$ & 614 & 13.2 & 0.2 & 0.3 \\
& 996 & 71.7 & 0.2 & 4.0 \\
\hline
\end{tabular}

etarias de 9-11 años $(n=355)$ y de 12-15 años $(n=259)$, se observó un aumento significativo en la prevalencia por antiVHA de $9.3 \%$ a $18.5 \%$, respectivamente (p. $<0.001$ ). Los marcadores de hepatitis B tienen una baja prevalencia en todas las poblaciones estudiadas.

La distribución por provincia de la prevalencia de anti-VHA y anti-HBc en la población pre-escolar se muestra en el Cuadro 3. Los resultados revelaron poca fluctuación en la prevalencia de los dos marcadores entre provincias, excepto para el anti-VHA que en Guanacaste muestra una prevalencia 2-3 veces mayor.

En el grupo de escolares se analizaron los datos demográficos disponibles y los resultados se muestran en el Cuadro 4. Se evidencia que la prevalencia de anti-VHA es muy inferior en los niños que asisten a las escuelas privadas en comparación con las públicas (6.8\% versus $19.5 \%)$.

El nivel de educación y ocupación de los padres también reveló una diferencia significativa, mostrando prevalencias más 
Cuadro 3: Prevalencia de marcadores infecciosos en preescolares $<7$ años por provincia

\begin{tabular}{|c|c|c|c|c|c|}
\hline \multirow[b]{2}{*}{ Provincia } & \multirow[b]{2}{*}{$\mathrm{N}^{\circ}$} & \multicolumn{2}{|c|}{ Anti-VHA } & \multicolumn{2}{|c|}{ Anti-HBc } \\
\hline & & Pos. & $\%$ & Pos. & $\%$ \\
\hline San José & 327 & 16 & 5 & 6 & 2 \\
\hline Alajuela & 126 & 8 & 6 & 8 & 6 \\
\hline Cartago & 62 & 0 & - & 1 & 2 \\
\hline Heredia & 55 & 3 & 6 & 1 & 2 \\
\hline Guanacaste & 91 & 19 & 21 & 2 & 2 \\
\hline Puntarenas & 127 & 11 & 9 & 4 & 3 \\
\hline Limón & 86 & 2 & 2 & 2 & 2 \\
\hline
\end{tabular}

\section{Cuadro 4: Prevalencia de marcadores infecciosos relacionados con datos demográficos en escolares de 9-15 años. San José, 1997}

\begin{tabular}{lllll}
\hline $\begin{array}{l}\text { Anti-VHA } \\
\text { Calidad }\end{array}$ & Total & Pos. & $\%$ & $\mathrm{p}$ \\
\hline Escuela pública & 307 & 60 & 19.5 & \\
Escuela privada & 307 & 21 & 6.8 & $\mathrm{p}<0.001$ \\
$\begin{array}{l}\text { Escolaridad del jefe de familia } \\
\quad\end{array}$ & & & \\
$\quad$ Secundaria incompleta & 141 & 34 & 24.1 & \\
$\quad$ Secundaria completa & 432 & 34 & 7.9 & $\mathrm{p}<0.001$ \\
$\begin{array}{l}\text { Ocupación del jefe de familia } \\
\quad \text { Profesional }\end{array}$ & 272 & 21 & 7.7 & \\
$\quad$ Comercio & 106 & 6 & 5.6 & \\
$\quad$ Otro* & 164 & 39 & 23.8 & $\mathrm{p}<0.003$ \\
\hline
\end{tabular}

*Sin capacitación técnica o profesional.

bajas de anti-VHA en los escolares con padres de niveles de educación y ocupación superiores (profesional y comercio).

El Cuadro 5, muestra la relación de los datos demográficos entre la población adulta. En los grupos de estudiantes y funcionarios se observan prevalencias muy altas en la población de $>30$ años, fluctuando entre $89 \%$ y 95\%. También es importante observar que los estudiantes $<30$ años tuvieron una prevalencia más baja que en los funcionarios $<30$ años (49\% versus $71 \%$ ).

\section{Discusión}

Las hepatitis A y B son endémicas en Costa Rica con prevalencias altas y bajas, respectivamente.

En un estudio realizado por el CIIAM en 1981, se encontró que en grupos de niños de 5-6 años de un área rural (San Ramón y Palmares), con alta y baja condición socioeconó-
Cuadro 5: Prevalencia de marcadores infecciosos relacionados con datos demográficos. UCR, 1994

\begin{tabular}{lccc}
\hline \multirow{2}{*}{ Población* } & \multirow{2}{*}{$\mathrm{N}^{\circ}$} & \multicolumn{2}{c}{ Anti-HAV } \\
& & $\mathrm{N}^{\circ}$ & $\%$ \\
\hline Estudiantes & 442 & 238 & 54 \\
Total & 399 & 195 & 49 \\
$<30$ años & 43 & 41 & 95 \\
$>30$ años & & & \\
Funcionarios & 271 & 235 & 87 \\
Total & 65 & 46 & 71 \\
$<30$ años & 206 & 184 & 89 \\
$>30$ años & & & \\
\hline *Unicamente se obtuvo información de 713 personas.
\end{tabular}

mica la prevalencia de anti-VHA fue de $11 \%$ versus $90 \%$, respectivamente. Sin embargo, el promedio de prevalencia en este mismo grupo de niños fue de $33 \%$ que contrasta con un $7 \%$ en niños $<7$ años del presente estudio. ${ }^{32,33}$ Esta marcada diferencia puede ser debida a mejoras en las condiciones de la población, las cuales han ido sucediendo paulatinamente, como una mayor cobertura de agua potable y letrinización. ${ }^{34}$

A pesar de estas mejoras, la transmisión de VHA según este estudio, sigue asociada a condiciones socioeconómicas de acuerdo con nuestros resultados. También se observó un aumento de la presencia de anti-VHA entre los escolares conforme aumenta la edad. Por otra parte, la prevalencia en estudiantes universitarios $<30$ años fué mucho menor que en la década de los 70's. ${ }^{11}$ De acuerdo con los resultados de este estudio, en la población de niños, Costa Rica pasó de un país de alta prevalencia de VHA a tener una prevalencia intermedia, con tendencia a seguir bajando. Probablemente, éste sea un cambio que ocurrió recientemente. Observaciones similares han sido reportadas en otros países como Argentina, Brasil, Chile, Guyana Francesa y Asia. ${ }^{35-40}$

Actualmente, se encuentran países con prevalencias bajas, intermedias y altas para la hepatitis B en la América Latina. ${ }^{23,41,42}$ Según estas observaciones y estudios previos, Costa Rica se podría clasificar como un país con prevalencias bajas, excepto en la región de San Isidro del General, en donde se presentan prevalencias intermedias. Debido a las bajas prevalencias de HBsAg encontradas en el presente estudio, no se pudo establecer una correlación entre las variables de riesgo y los datos disponibles. Aún cuando no se encontraron diferencias significativas entre las poblaciones analizadas, se observaron más portadores en los niños $<7$ años (Cuadro 2), lo que puede sugerir un mecanismo de transmisión horizontal entre ellos. ${ }^{25,42}$ La infección por VHB, como ya es conocido, parece estar asociada al sexo masculino. ${ }^{2}$ Esto se evidencia en el estudio de adultos, con una prevalencia de $6,9 \%$ en hombres y $2,3 \%$ en mujeres con anti-HBc, el marcador de uso para determinar infecciones presentes o pasadas por VHB. 
En la actualidad en Costa Rica se están implementando medidas de prevención y control para VHB a través de un programa de vacunación para todos los recién nacidos y diferentes grupos de riesgo, personal hospitalario, poblaciones promiscuas, etc. Recientemente, se realizó un estudio de evaluación de respuesta a la vacuna en recién nacidos en la zona de prevalencia intermedia obteniéndose resultados similares a lo reportado en la literatura, con una respuesta superior. ${ }^{43}$

La vacuna para hepatitis A está disponible, pero todavía tiene un costo muy elevado para campañas de vacunación masiva. En vista de los resultados presentados en este estudio se debe hacer conciencia en la población sobre el cambio de patrones de prevalencia y los mecanismos de riesgo de infección por VHA, especialmente entre los grupos de adolescentes y adultos en donde el curso de la enfermedad puede tener manifestaciones severas.

\section{Agradecimientos}

Nuestro especial agradecimiento a la Srta. Sofía Castillo R., Depto. de Archivo, Oficina de Salud, UCR, al Dr. Luis Tacsan, Ministerio de Salud, a la Compañía Smith Kline Beecham, y a todas aquellas personas que nos brindaron su colaboración para la conclusión de este estudio.

\section{Abstract}

Justification and objectives: Due to persistence of acute cases and outbreaks of viral hepatitis A and B in our country and the observation of an important increase of type A infections in the adult population, it was considered necessary to establish the prevalence of these two types of hepatitis in different age groups in our country.

Methods: Seroprevalence of viral hepatitis A (HAV) and viral hepatitis B (HBV), was studied in a population which included 873 pre-scholar children $<7$ years from a national nutritional survey in 1996; 614 scholar children 9-15 years from a national nutritional survey carried out by the University of Costa Rica during 1996 and 1997, and 996 samples from students and personnel from this same university collected during 1994. These samples were analyzed for total antibodies to HAV (anti-HAV total), HBV surface antigen (HBsAg) and total antibodies against core (anti-HBc total).

Results: The percentage of anti-HAV was established to be $6.8 \%, 13.2 \%$ and $71.7 \%$ for the pre-scholar children, scholars and adults, respectively, with an increasing prevalence related to age. The presence of antibodies in the "scholar group" was associated with education level of the parents and their socioeconomic status; being higher for parents with a lower education level (24\% with incomplete high school versus $7.6 \%$ with higher education level) and among parents without technical or professional training (16\% versus $8.7 \%$ in the parents with higher education level). Also, it was a difference between the presence of anti-HAV among students from public schools (19.5\%) versus private schools (6.9\%). In the prescholar children, which was the only group with geographic statistical significance, the province of Guanacaste showed a 2-3 times higher risk to have HAV antibodies (21\%) than in children from other provinces. This is in concordance with data related to low socio-economical status, lack of potable water and poor sanitary conditions.

The study showed a prevalence of HBV markers to be $0.5 \%$, $0.2 \%$ and $0.2 \%$ for $\mathrm{HBsAg}$, and $2.8 \%, 0.3 \%$ and $4.0 \%$ for anti-HBc in pre-scholars, scholars and adults, respectively. There was no relationship between the socioeconomic status or age, with these prevalences; but the anti-HBc was found to be higher in men (6.9\% in men and $2.3 \%$ in women).

Conclusions: When comparing the data obtained in earlier decades in Costa Rica for these two types of hepatitis, it was found that hepatitis B continues to have a similar epidemiological pattern with a low prevalence, while hepatitis A changed the pattern during childhood from a high prevalence to an intermediate to low prevalence. This change requires an adequate intervention to avoid epidemic outbreaks and increase in the number of severe cases in the young and adult population.

\section{Referencias}

1. Hollinger FB, and Ticehurst JR. Hepatitis A Virus. En :Fields BN, Knipe DM, Peter MH, et al., eds. Virology. 3ra ed. Vol. 1: Part II. Specific Virus Families. Picornaviridae. Philadelphia. New York. Lippincott-Raven Publishers. 1996; 735-782.

2. Hollinger FB. Hepatitis B Virus. En :Fields BN, Knipe DM, Peter $\mathrm{MH}$, et al., eds. Virology. 3ra ed. Vol. 2: Part II. Specific Virus Families. Hepadnaviridae. Philadelphia. New York. Lippincott-Raven Publishers. 1996; 2738-2807.

3. Feinstone SM, Kapikian AZ, Purcell PH. Hepatitis A. Detection by immune electron microscopy of a virus like antigen association with acute illness. Science 1973; 182:1026-1028.

4. Raymond SK. Hepatitis A. Lancet 1998; 34: 1643-1649.

5. Rosemblum LS, Villarino ME, Nainan OV, et al. Hepatitis A outbreak in a neonatal intensive care unit: risk factors for transmission and evidence of prolonged viral excretion among preterm infants. J. Infect. Dis. 1991; 164: 476-482.

6. Pañella H., Bayas JM, Maldonado R, Caylá JA, Vilella A, Sala C, et al. Brote epidémico de hepatitis A relacionado con una guardería. Gastroenterol y Hepatol 1998; 21: 319-323.

7. Chudy M, Budek I, Keller-Stanislawski B, McCaustland KA, Neidhold S, Robertson B, et al. A new cluster of Hepatitis A infection in hemophiliacs traced to a contaminated plasma pool. J Med Virol 1999; 57 : 91-99.

8. Soucie JM, Robertson BH, Bell BP, McCaustland KA, Evatt, BL. Hepatitis A virus infections associated with clotting factor concentrate in the United States. Transfusion 1998; 38: 573-579.

9. Robertson BH, Jansen RW, Khanna B, et al. Genetics relatedness of hepatitis A virus strains recovered from different geographical regions. J Gen Virol 1992; 73: 1365-1377. 
10. Centers for Disease Control and Prevention (CDC). Recomendations and Reports. Prevention of Hepatitis A Through Active or Passive Immunization. Recomendations of the Advisory Committee on Immunization Practices (ACIP). U.S. Department of Health and Human Services. Public Health Services. Atlanta Georgia. MMWR 1999; 48 (RR-12).

11. Villarejos VM, Serra J, Anderson-Visoná K, Mosley J. Hepatitis A in households. Am J Epidemiol 1982; 115: 577-586.

12. Dal-Ré R, García-Corbeira P and García de Lomas J. A large percentage of the spanish population under 30 years of age is not protected against hepatitis A. J Med Virol 2000; 60: 363-366.

13. Cruells MR, Mescia G, Gaibisso R, Ramírez M, Gutiérrez M, Kohen $\mathrm{S}$, et al. Estudio epidemiológico de los virus de la hepatitis A y E en diferentes poblaciones de Uruguay. Gatroenterol y Hepatol 1997; 20: 295-298.

14. Sathar MA, Soni PN, Fernandes-Costa FJTD, Wittenberg DF, and Simjee AE. Racial differences in the seroprevalence of hepatitis A virus infection in Natal/KwaZulu, South African J Med Virol 1994; 44: $9-12$.

15. McMahon BJ, Beller M, William J, et al. A program to control of outbreaks of hepatitis A in Alaska by using an inactivated hepatitis A vaccine. Arch Pediatr Adolesc Med 1996; 150: 733-739.

16. Sagliocca L, Amoroso P, Stroffolini T, Adamo B, Tosti ME, Lettieri G, et al. Efficacy of hepatitis A vaccine in prevention of secundary hepatitis A infection: a randomised trial. Lancet 1999; 353: 1136-1139.

17. Alter MJ, Hadler SC, Margolis HS, et al. The Changing epidemiology of Hepatitis B in the United States: need for alternative vaccination strategies. JAMA. 1990; 263: 1218-1222.

18. Davison F, Alexander GJM, TrowbridgeR, Fagan EA, Williams R. Detection of hepatitis B virus DNA in spermatozoa, urine, saliva and leucocytes of chronic HBsAg carriers. Hepatology 1987; 4: 37-44

19. The program of the World Health Organization in the international surveillance of viral hepatitis B. Am J Med Sci 1975; 270: 283-285.

20. Forns X y Ampurdarnés S. Clínica de las hepatitis virales, hepatitis aguda, hepatitis crónica, carcinoma hepatocelular. Enfermedades Infecciosas y Microbiología Clínica 1995; 13 (supl.1): 71-83.

21. Rodrigo L, Rodríguez M, Linares A. Etiología y epidemiología de la hepatitis crónica. Gastroenterol y Hepatol 1994; 17:50-59.

22. Centers of Disease Control and Prevention (CDC). Final 1993 report of specified notifiable diseases. U.S. Department of Health and Human Services. Public Health Services. Atlanta Georgia MMWR 1994, 43: 597-603.

23. Tanaka J. Hepatitis B epidemiology in Latin America. Vaccine 2000; 18 (Suppl 1): S17-S19.

24. Sagliocca L, Stroffolini T, Amoraso P, Manzillo G, Ferrigno L, Converti F, Palumbo F, Izzo E, and Mele A. Risk factors for acute hepatitis B: a case-control study. J V Hepatitis 1997; 4:63-66.

25. Visoná KA, Eduarte CE, Zamora E y Salazar LM. Estudio epidemiológico de las hepatitis virales en San Ramón y Palmares de 19721985. Acta Med Costarric 1989; 33: 69-77.

26. Esquivel J, Fernández L, Hevia F, Martén A, Mora J y Salóm I. Diagnóstico y prevención de las hepatitis virales. En: Esquivel JM. 1 Hepatitis. 2 Hepatitis Viral Humana, 1ra. ed EDENASSS-CCSS. San José. Costa Rica. 1990; 1-22.

27. Wu JS, Hwang L-Y, Goodman KJ. Hepatitis B vaccination in high-risk infants: 10-year follow-up. J Infect Dis 1999; 179: 1319-1325.
28. Harpaz R, McMahon BJ. Elimination of new chronic hepatitis B virus infection: results of the Alaska immunization program. J Infect Dis 2000; 181: 413-418.

29. Decker RH, Overby LR, Ling CM, Frosner G, Deinhardt F, Boggs J. Serologic studies of transmission of hepatitis A in humans. J Infect Dis 1979; 139: 74-82.

30. Visoná KA. Viral Hepatitis in a Developing Country. Ph.D Thesis, 1992; University of Copenhagen, Denmark.

31. Arauz R. P., Norder H., Visona K. A., and Magnius OL. Genotype F Prevails in HBV Infected Patients of Hispanic Origin in Central America and May Carry The precore Stop Mutant. J Med Virol 1997; 51: 305-312.

32. Serra J, Visoná KA, Villarejos VM, Castillo C, Serrano AM. Inmunidad pasiva contra la hepatitis A y B por anticuerpos maternos. VII Jornadas Latinoamericanas y I Congreso Nacional de Hepatología. San José, Costa Rica 1981; octubre 21-24.

33. Villarejos VM, Visoná KA, Serra J, Mosley JW. Observaciones sobre la ocurrencia de infecciones por el virus de hepatitis tipo A en un área endémica. VII Jornadas Latinoamericanas y I Congreso Nacional de Hepatología. San José, Costa Rica, 1981; octubre 21-24.

34. Ministerio de Planificación Nacional y Política Económica(MIDEPLAN). Area de Análisis del Desarrollo: Unidad de Desarrollo Social. Sistema de Indicadores Sobre Desarrollo Sostenible (SIDES). Principales indicadores sociales de Costa Rica. 1997; Serie N², 81-105.

35. Daruich JR, Rey JA, Pinchuk L, Igartúa EB, Zeilicoff R, Michellini J, et al. Prevalencia de anti HAV en población general en Buenos Aires, Argentina. XV Congresso da Associaçâo Latino-Americana para o Estudio do Fígado-ALEF. G. E. D. 1998; 17 (supl.): 57.

36. Pinto PTA, Müller AM, Vieira MTM, Nóbrega ICP, Corrêa VLF. Soroprevalencia da hepatite A em serviço de medicina de familia. XV Congresso da Associaçâo Latino-Americana para o Estudio do FígadoALEF. G. E. D. 1998; 17 (supl.): 57.

37. Lagos R, Potin M, Muñoz A, Abrego P, Martin O S, Ureta A M, et al. Anticuerpos séricos contra el virus de la hepatitis A entre personas de niveles socioeconómicos medios y bajos en un área urbana de Santiago, Chile. Rev Med Chil. 1999; 127: 429-436.

38. Talarmin A, Kazanji M, Cardoso T, Pouliquen J-F, Sankale-Suzanon J, Sarthou J-L. Prevalence of antibodies to hepatitis A, C, and E viruses in different ethnic groups in French Guiana. J Med Virol 1997; 52: 430-435.

39. Mayorga PO, Morales W, Paniagua M and Strannegard O. Prevalence of Antibodies to Hepatitis A, B, C, and E Viruses in a Healthy Population in León, Nicaragua.Am J Trop Med Hyg 1996; 55 17-21.

40. Kunasol P, Cooskley G, Chan V F, Isahak I, John J, et al. Hepatitis A virus: declining seroprevalence in children and adolescents in southeast Asia. Southeast Asian J Trop Med Public Health 1998; 29: 255-262.

41. Galvao-Alves J, Tyll J, Brum A, Rzetelna H, Mello MAS, Moneró F, et al. Incidência de HBsAg em 90632 doadores de sangue. XV Congresso da Associaçâo Latino-Americana para o Estudio do Fígado-ALEF. G. E. D. 1998; 17 (supl.): 59.

42. Cabezas C, Reátegui J, Suaréz M, Romero DG, Carrillo C, Vallenas F, et al. Hiperendemicidad de hepatitis virales $\mathrm{B}$ y delta en pueblos indígenas de la amazonia peruana. XV Congresso da Associaçâo Latino-Americana para o Estudo do Fígado-ALEF. G. E. D. 1998; 17 (supl.): 61.

43. Calvo N. Evaluación serológica de la inmunización infantil contra el virus de hepatitis B en Pérez Zeledón. Costa Rica, 1999. Universidad Nacional Autónoma, Sistema de Posgrado. Posgrado Regional en Ciencias veterinarias Tropicales. Mención en Epidemiología, Heredia, noviembre 19, 2000. 